\title{
A SIMULATION MODEL FOR A SAME DAY CARE FACILITY AT A UNIVERSITY HOSPITAL
}

\author{
Wafik H. Iskander \\ D. Mark Carter \\ Department of Industrial Engineering \\ West Virginia University \\ Morgantown, WV 26506-6101
}

\begin{abstract}
A model was developed to simulate the operations of the Same Day Care Unit (SDCU) at a major medical institution, and to evaluate the system performance for different alternatives, under various patient load conditions. The model was used to successfully identify the facility needs at the SDCU in order to optimize patient care and accommodate projected growth in patient volumes.
\end{abstract}

\section{INTRODUCTION}

During the mid to late 80 's, there was a strong shift from inpatient to outpatient medical care. Rapid health care advances and cost containment pressures have been breaking new ground in transforming inpatient services into outpatient services. During this time period, new facilities were being planned and constructed for the medical center at West Virginia University. These facilities include a 100 million dollar tertiary care hospital, joined by a 23 million dollar outpatient care facility that houses a new Ambulatory Care Center (ACC). At the time of the original planning of the new facilities, the Same Day Care Unit (SDCU) was relatively new, and all industry projections greatly underestimated its growth. Important decisions had to be made in the process of moving the SDCU into the new complex. In the relatively short period of time from which the facilities were planned, designed and construction begun, enormous growth was taking place in SDCU patient volumes and types. Proper decision timing and accuracy were critical to ensure adequate customer service, safe conditions, and reasonable costs for facility modifications.

The SDCU was to be housed in the comprehensive outpatient facility, which was still under construction.
This facility is connected to the main hospital, and was designed to handle procedures such as endoscopy, cystoscopy, minor surgery, and recovery from these procedures, as well as preparation and recovery from surgery performed in the main hospital operating rooms. Several decisions had to be made about the size of the waiting area, number of beds needed in the recovery area, and adequacy of the facilities used for the different procedures.

To evaluate the adequacy of the current ACC space and facilities planned for the SDCU, a simulation model was developed and implemented. The model was used to analyze the SDCU operations and to define its needs under the current conditions as well as under different modes of operation. Of particular interest was the effect that growth in volume would have. Application of simulation to the health field is not new, and many examples can be found in the literature (Currie et al. 1984 \& Kumar and Kapur 1989). Description of the model developed and a summary of some of the results produced are given below.

\section{THE SAME DAY CARE UNIT}

The SDCU and the flow of patients associated with it can best be described with the help of figures 1 and 2 . The initial design of the SDCU includes a waiting area where patients and their families wait, two changing rooms, one for males and the other for females, and a preparation/recovery area where patients undergo a preparation process before some of the operations, and recover after their operations and procedures. The preparation/ recovery area was designed to accommodate 14 beds and 4 lounges.

Under the initial design, the SDCU is connected to the following facilities, which are housed in the ACC building and are considered as part of the simulation 


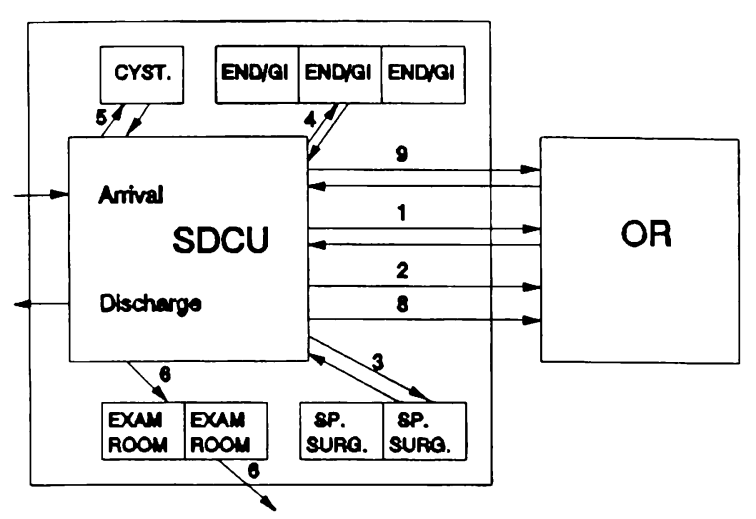

Figure 1: SDCU System

system developed: A Cystoscopy room, three Endoscopy/Gastroenterology (GI) rooms, two rooms for outpatient surgery, and two examination rooms that are used by anesthesiologists for pre-operation testing. The SDCU is also connected to the operating rooms (OR) which are housed in the adjacent main hospital.

Patients going through the SDCU were classified in one of the following 9 categories:

1. Same day care patients - These patients arrive at the SDCU, change clothes, go through the preparation process, and then are sent to the OR. They return later in the same day to the SDCU for a period of recovery, and then are discharged.

2. Morning admission patients (AM-IN) - These are similar to category one, except that they get admitted to the main hospital after their operation instead of returning to the SDCU.

3. Outpatient surgery - Patients arrive at the SDCU, change but do not go through the preparation process, go for their procedure in one of the two surgery suites, recover in the SDCU, and then get discharged.

4. ENDO/GI - Same as 3, but patients go to one of the $3 \mathrm{ENDO} / \mathrm{GI}$ rooms instead of the surgery rooms.

5. CYSTO - Same as 3 and 4 , but patients go to the Cystoscopy room.

6. PRE-OP - These patients arrive at random to the SDCU, often following a clinic visit in the adjacent clinic area. They go directly to one of the two anesthesia examination rooms without changing and without preparation, and then leave directly without coming back to the SDCU.

7. Cancellation - A patient slot is reserved, but either the patient or the physician cancels, or the patient does not show.

8. Same day care patients changed to admission Same as category 1 but patients are admitted due

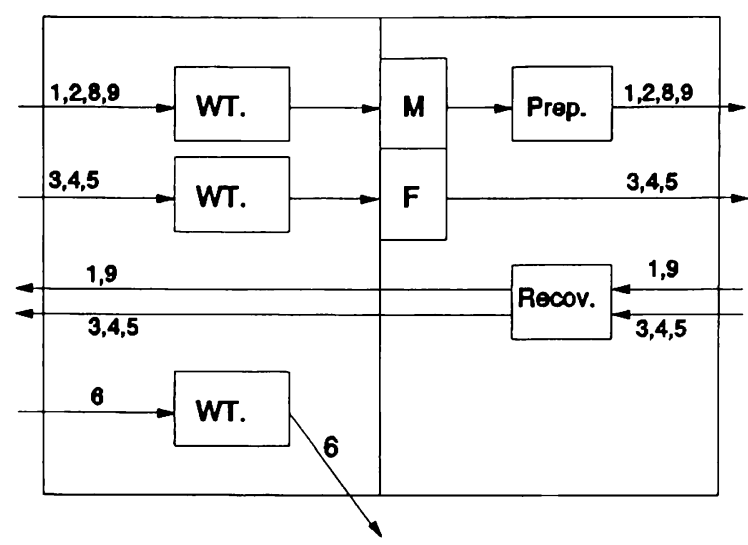

Figure 2: Flow of Patients in the SDCU

to complications in their operation. In this case, patients do not return to the SDCU after their operation.

9. Admission changed to same day care patients Same as category 2 but due to ease/success of the procedure, patients are not admitted but instead sent back to the SDCU for recovery and discharge. The flow of patients for all the above cases are shown in figures 1 and 2.

One may question the difference between categories 1 and 9 or between categories 2 and 8 . It is true that the patients flow is the same in each of these cases regardless of the original classification, however the waiting process for their relatives is different. If a patient is scheduled for admission, his relatives do not stay in the SDCU waiting area after he leaves to the $\mathrm{OR}$. On the other hand, relatives do wait for a "same day care" patient until he is discharged or until his status is changed to admission. The data indicated that patients changed categories often enough that the changes in number of relatives waiting in the waiting area needed to be considered.

The OR patients (categories 1, 2, 8, and 9) go to one of the following services:

1. Ear Nose and Throat (ENT)

2. Endocrinology

3. Orthopedics

4. Gynecology

5. Ophthalmology

6. Others (Cardiology, Oral Surgery, Neuro Surgery, and Plastic Surgery)

7. Oncology

8. Urology

9. General Surgery

The duration of the operation, as well as many other parameters, depend on the service rendered to the patient as described in the next section.

The main questions that needed to be answered include the following: 
1. How big should the waiting area be in the SDCU?

2. How many beds and lounges are needed in the preparation/recovery area of the SDCU?

3. Is one changing room sufficient for each gender?

4. Are the facilities provided for all services adequate? Are they over or under-utilized? The OR facilities are not included here as they are not part of the new ACC under design.

5. If the patients are considered alone, without accompanying relatives, how big should their waiting area be? This question was considered in order to investigate the option of sending waiting relatives to another location.

To answer these questions, the simulation model was developed and the operations at the SDCU were simulated under different conditions as described below.

\section{THE SIMULATION MODEL}

To develop the simulation model, a massive amount of data was obtained from a detailed record keeping system maintained by the hospital. After thorough analysis of the data, the following conclusions were made and later used as assumptions in the model:

1. The number of $O R$ cases that come every day through the SDCU (categories 1, 2, 8, and 9), and the statistical distribution for their inter-arrival times depend on the day of the week, with Mondays and Thursdays being the busiest. Hence data were considered only for Mondays and Thursdays, each individually, as details of the cases can be different between the two days.

2. Distributions of the OR cases to the different services are different between Mondays and Thursdays, but they follow the same pattern within each of the two days.

3. Statistical distributions for waiting time for the $O R$ cases depend on the day of the week but not on the service provided. The only exception is for the oncology patients (service code 7) who are anticipated and expedited through the system and end up waiting for about 5 minutes only.

4. An effective way to simulate the $O R$ cases is to generate individual arrivals at random according to the appropriate distribution for the day under consideration, assign their service code, again using the appropriate distribution for the day, and then assign all other parameters for the case.

5. For OR cases, both patient's gender and category $(1,2,8$, or 9$)$ are functions of the service rendered, but not of the date or day of the week.

6. Statistical distributions for both operation time and recovery time for the $O R$ patients depend on the service provided, but not on date, day of week, or gender.

7. Parameters of all cases other than OR (categories 3 through 6) do not change between the different days of the week.

The flow of transactions (patients and accompanying relatives) through the simulation system is summarized below.

\subsection{Operating Room Patients}

These patients start arriving every day at 6:00 am except on Mondays when they start arriving at 7:00 am. The number of arrivals per day is uniformally distributed (between 17 and 26 on Mondays). The distribution of inter-arrival time depends on the day of the week. An example is given in figure 3 for Mondays.

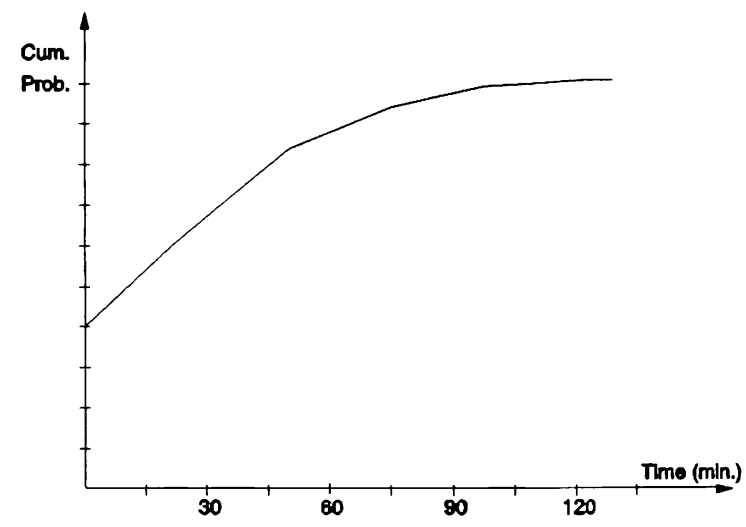

Figure 3: Distribution of O.R. Inter-arrival Time for Mondays

A service code and a waiting time are then generated for the patient. Distributions of these codes and times depend on the day of the week. Examples are given in Table 1 and figure 4. Depending on the service code assigned to the patient, the following parameters are determined: Gender, category $(1,2,8$, or 9), procedure time, and recovery time. Examples of the distributions used are given in Table 2 and figures 5 and 6. A number of people between 0 and 5 accompany the patient and arrive with $\mathrm{him} / \mathrm{her}$ at the same time. Distribution of this number is given in Table 3. After waiting, the patient goes to the appropriate changing room where he/she spends between 2 to 3 minutes and then occupies a bed in the preparation/recovery area where he/she spends between 30 and 35 minutes. However, Oncology patients do no go through the preparation procedure. For simulation purposes, it was assumed that an unlimited number of beds is available. For patients who are not scheduled to return to the SDCU in the 
Table 1: Distribution of O.R. Service Codes

\begin{tabular}{||l|c|c|c|c|c|c|c|c|c||}
\hline \hline Service & 1 & 2 & 3 & 4 & 5 & 6 & 7 & 8 & 9 \\
\hline Prob. & .22 & .00 & .18 & .07 & .16 & .19 & .05 & .11 & .02 \\
\hline
\end{tabular}

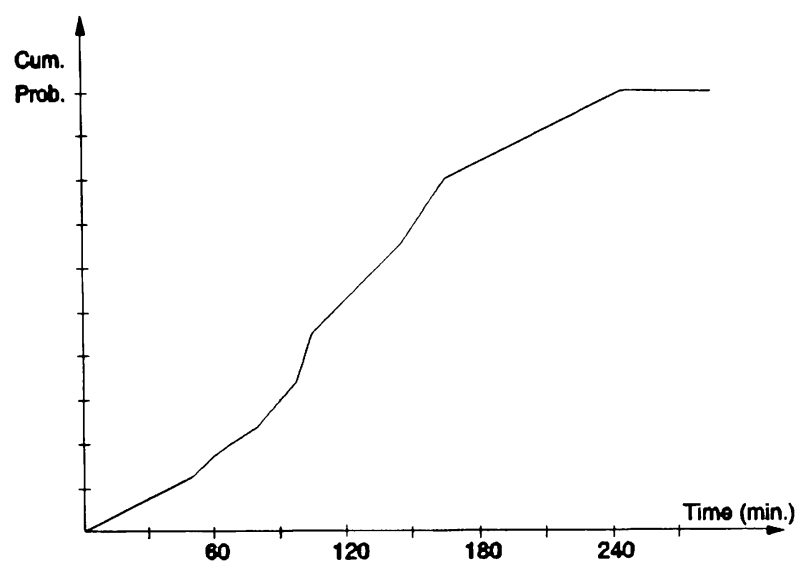

Figure 4: Distribution of O.R. Waiting Time for Mondays

Table 2: Distribution of Gender for Orthopedics

\begin{tabular}{|l|l|l|}
\hline Gender & M & F \\
\hline Probability & .63 & .37 \\
\hline
\end{tabular}

same day (categories 2 and 9) the accompanying people leave the waiting area as soon as these patients are taken to the changing rooms. Upon completion of the operation/procedure, the following takes place: Category 2 patients are sent out of the system. Category 8 patients are also sent out of the system, but their relatives leave the SDCU waiting area only at this time, after they learn of the patient's status change. Category 1 and 9 patients return to the SDCU preparation/recovery area. Relatives of category 1 patients have never left the SDCU waiting area, however those of category 9 patients will come back to the waiting area at this stage as they learn of the patient's change of status. The patient then waits on a bed in the SDCU preparation/recovery area until he recovers and then leaves, together with his relatives.

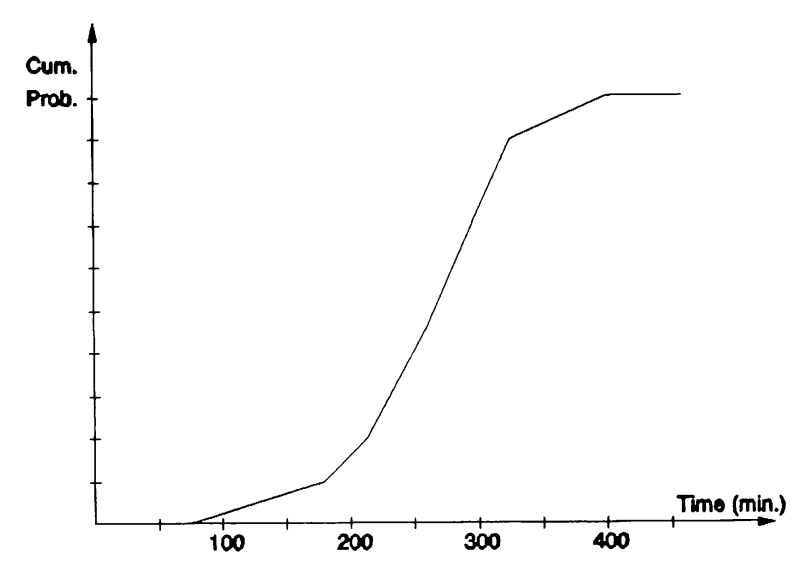

Figure 5: Distribution of Procedure Time for Orthopedics

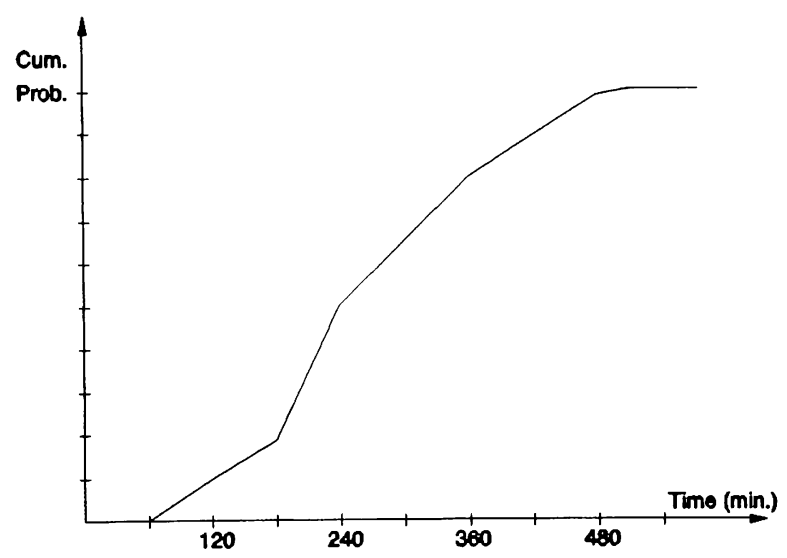

Figure 6: Distribution of Recovery Time for Orthopedics

\subsection{Pre-Op Patients}

These patients start arriving at 7:30 am according to an exponential inter-arrival time with a mean of 24 minutes. They keep arriving until their total reaches a number determined from the distribution shown in figure 7. These patients are accompanied by one person ( $80 \%$ of the time), 2 persons $(15 \%)$, or arrives 
alone (5\%). After waiting between 15 and 25 minutes, they go directly to one of the two examination rooms where they spend between 15 and 25 minutes for their examination and then leave directly together with their relatives.

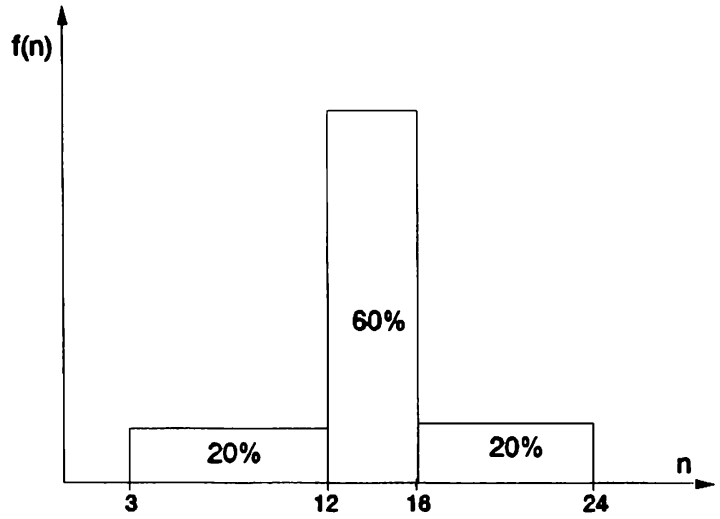

Figure 7: Distribution of Number of Arrivals - Pre Op

\subsection{Outpatient Surgery Patients}

A total of 10 to 12 patients come every day starting at 7:00 am according to an exponential inter-arrival time with a mean of 40 minutes. The number of accompanying persons follows the distribution given in Table 3.

Table 3: Distribution of Number of Persons Accompanying Patient

\begin{tabular}{||l|c|c|c|c|c||}
\hline \hline Number & 0 & 1 & 2 & 3 & 4 \\
\hline Probability & .10 & .70 & .10 & .06 & .04 \\
\hline
\end{tabular}

About 50 percent of the patients are male. After waiting 24 to 36 minutes, the patient is taken to the appropriate changing room where he/she spends 2 to 3 minutes, and then goes directly to one of the two outpatient surgery rooms. Surgery time is determined from the distribution shown in figure 8. Upon completion of the surgery, the room is prepared in about 15 minutes before it can receive the next patient, and the current patient is taken back to the SDCU Preparation/Recovery area where he spends 54 to 66 minutes for recovery on a lounge and then leaves, accompanied by his relatives. If no lounge is available, the patient recovers on a bed, however for the simulation purpose an infinite number of lounges was assumed to be available.

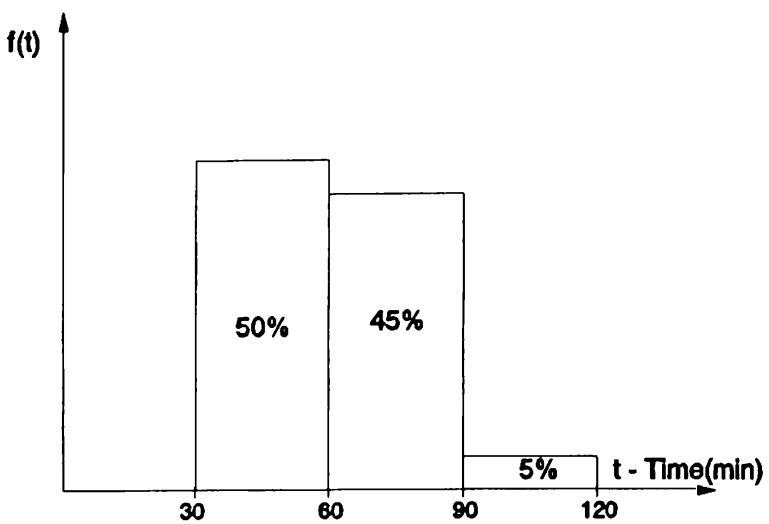

Figure 8: Distribution of Surgery Time for Outpatient Surgery

\subsection{Cystoscopy Patients}

Seven patients are scheduled to arrive each day at the following times: 7:00, 8:15, 9:30, and 10:45 am, and $12: 30,1: 45$, and $3: 00 \mathrm{pm}$. All the patients usually show up, and they come about 0 to 10 minutes early, accompanied by a number of relatives according to the distribution given in Table 3 . About 50 percent of the patients are male. After waiting 30 to 40 minutes, the patient is taken to the appropriate changing room where he/she spends 2 to 3 minutes, and then goes directly to the Cystoscopy room where he spends between 54 and 66 minutes for his procedure. After the procedure, the patient is taken back to a bed in the SDCU Preparation/Recovery area where he spends 54 to 66 minutes for recovery and then leaves with his relatives.

\subsection{Endo/GI Patients}

These patients go through the same steps as the Cystoscopy patients, except that 21 of these patients are scheduled to arrive every day starting at 7:00 am and every 20 minutes thereafter, and about 10 percent of these patients do not show. Needless to say, these patients go to one of the three Endo/GI rooms as opposed to the Cystoscopy room.

One may question the reason for having the patient waiting time as input to the model rather than output. This is due to the fact that patients coming into the SDCU do not usually wait for the facilities (eg. exam rooms, beds, etc.) but rather for personnel (eg. doctors, nurses, etc.), and these were not considered as part of the system with the assumption 
that their availability and level of service will not significantly change.

\section{SIMULATION OUTPUT}

The model was developed to run the simulation for a large number of days (eg. $n=100$ or 200 days) and to produce the following outputs for each half an hour period between 6:00 am and 8:30 pm.

1. An average for the $n$ simulated days for the maximum number of people waiting in the SDCU waiting area.

2. The maximum during the $\mathrm{n}$ simulated days of the maximum number of people waiting in the SDCU waiting area.

3. An average for the maximum number of beds required in the preparation/recovery area of the SDCU.

4. The maximum of the maximum number of beds required in the preparation/recovery area of the SDCU.

5. An average for the maximum number of patients waiting in the SDCU waiting area.

6. The maximum of the maximum number of patients waiting in the SDCU waiting area.

In addition, the following outputs were designed to be produced by the model:

7. Maximum number of lounges needed in the SDCU preparation/recovery area.

8. Utilization of each of the two changing rooms.

9. Utilization of each of the procedure and examining rooms (except for the OR)

10. Average waiting time of all people in the waiting area.

11. Average waiting time of patients in the waiting area.

12. Frequency distributions of the daily maximum number of beds needed in the preparation/recovery area, and of the maximum number of people waiting in the SDCU waiting area. These distributions were used to determine the percentage of the maximum daily requirements that are accommodated by specific designs.

\section{PROGRAM DESCRIPTION}

The simulation model was developed using GPSS-H. Several versions of the program were developed to cover different scenarios that were investigated. Sizes of the programs range between 200 and 230 GPSS blocks and between 370 and 460 statements. The program has six segments, a control segment and 5 other segments, one for each type of patients defined above. The control segment was used to initialize variables and statistics at the beginning of every day, and to collect statistics at the end of each half hour period between 7:00 am and 8:30 pm. Each of the other segments was used to control the flow of patients and their relatives through the system. In general, patients were simulated as transactions, and all the SDCU facilities were simulated as facilities, storages, and queues. The program was straight forward as the problem was very much suited to the application of GPSS.

\section{MODEL VALIDATION}

In order to validate the model, statistics were obtained from simulating various simplified scenarios and checked for their face validity. Sensitivity analyses were also performed while changing input variables and parameters and testing the effect on the results produced. The model performed well in all cases and was considered fit for implementation. The ultimate validation of the model came later, after its implementation and after the design recommendations were made, as the current, real life operations at the SDCU produce similar results to those predicted by the model.

\section{IMPLEMENTATION AND RESULTS}

The simulation model was used to investigate the system performance under nine different scenarios and modes of operation as described below:

1. All SDCU services accommodated in the ACC Existing demand levels.

2. Oncology and Pre-operation visits moved to other facilities - Existing demand levels.

3. Oncology visits moved - $20 \%$ growth in SDCU volume.

4. Oncology visits moved - $50 \%$ growth in SDCU volume.

5. Oncology and Pre-operation visits moved - $20 \%$ growth in SDCU volume.

6. Oncology and Pre-operation visits moved - $50 \%$ growth in SDCU volume.

7. All SDCU services accommodated in the ACC, but all discharges moved - Existing demand levels.

8. Oncology and Pre-operation visits, and recoveries requiring more than 3 hours moved - Existing demand levels.

9. Oncology and Pre-operation visits, and recoveries requiring more than 4 hours moved - Existing demand levels.

A summary of the results produced for Mondays is given in Table 4. An example of the maximum 
Table 4: Summary of Results

\begin{tabular}{|c|c|c|c|c|c|c|c|c|c|}
\hline Case & 1 & 2 & 3 & 4 & 5 & 6 & 7 & 8 & 9 \\
\hline Peak Bed Required & 18 & 18 & 19 & 21 & 18 & 22 & 12 & 14 & 14 \\
\hline $\begin{array}{l}\text { \% of Maximum Bed Requirement } \\
\text { Accommodated by } 12 \text { Beds } \\
\begin{array}{ll}14 \text { Beds } \\
16 \text { Beds }\end{array}\end{array}$ & $\begin{array}{l}58 \\
82 \\
97\end{array}$ & $\begin{array}{l}-- \\
-- \\
--\end{array}$ & $\begin{array}{l}29 \\
63 \\
90\end{array}$ & $\begin{array}{l}14 \\
39 \\
71\end{array}$ & $\begin{array}{l}-- \\
-- \\
--\end{array}$ & $\begin{array}{l}-- \\
-- \\
--\end{array}$ & $\begin{array}{l}100 \\
100 \\
100\end{array}$ & $\begin{array}{c}94 \\
100 \\
100\end{array}$ & $\begin{array}{r}99 \\
100 \\
100\end{array}$ \\
\hline Peak Waiting Room Occupancy & 100 & 98 & 127 & 121 & 108 & 117 & 51 & 56 & 84 \\
\hline $\begin{array}{l}\% \text { of Maximum Waiting Room } \\
\text { Occupancy Accommodated } \\
\text { by } 28 \text { Spaces } \\
50 \text { Spaces } \\
75 \text { Spaces } \\
100 \text { Spaces }\end{array}$ & $\begin{array}{c}0 \\
0 \\
39 \\
100\end{array}$ & $\begin{array}{l}-- \\
-- \\
-- \\
--\end{array}$ & $\begin{array}{c}0 \\
0 \\
19 \\
97\end{array}$ & $\begin{array}{c}0 \\
0 \\
10 \\
79\end{array}$ & $\begin{array}{l}-- \\
-- \\
- \\
--\end{array}$ & $\begin{array}{l}-- \\
-- \\
-- \\
--\end{array}$ & $\begin{array}{c}0 \\
19 \\
99 \\
100\end{array}$ & $\begin{array}{c}0 \\
7 \\
92 \\
100\end{array}$ & $\begin{array}{r}0 \\
11 \\
99 \\
100\end{array}$ \\
\hline Average Maximum Bed Requirements & 10.5 & 10 & 12 & 13 & 11 & 13 & 7 & 8 & 8 \\
\hline $\begin{array}{l}\text { Average Maximum Waiting } \\
\text { Room Requirements }\end{array}$ & 72 & 67 & 77 & 83 & 73 & 82 & 51 & 56 & 56 \\
\hline
\end{tabular}

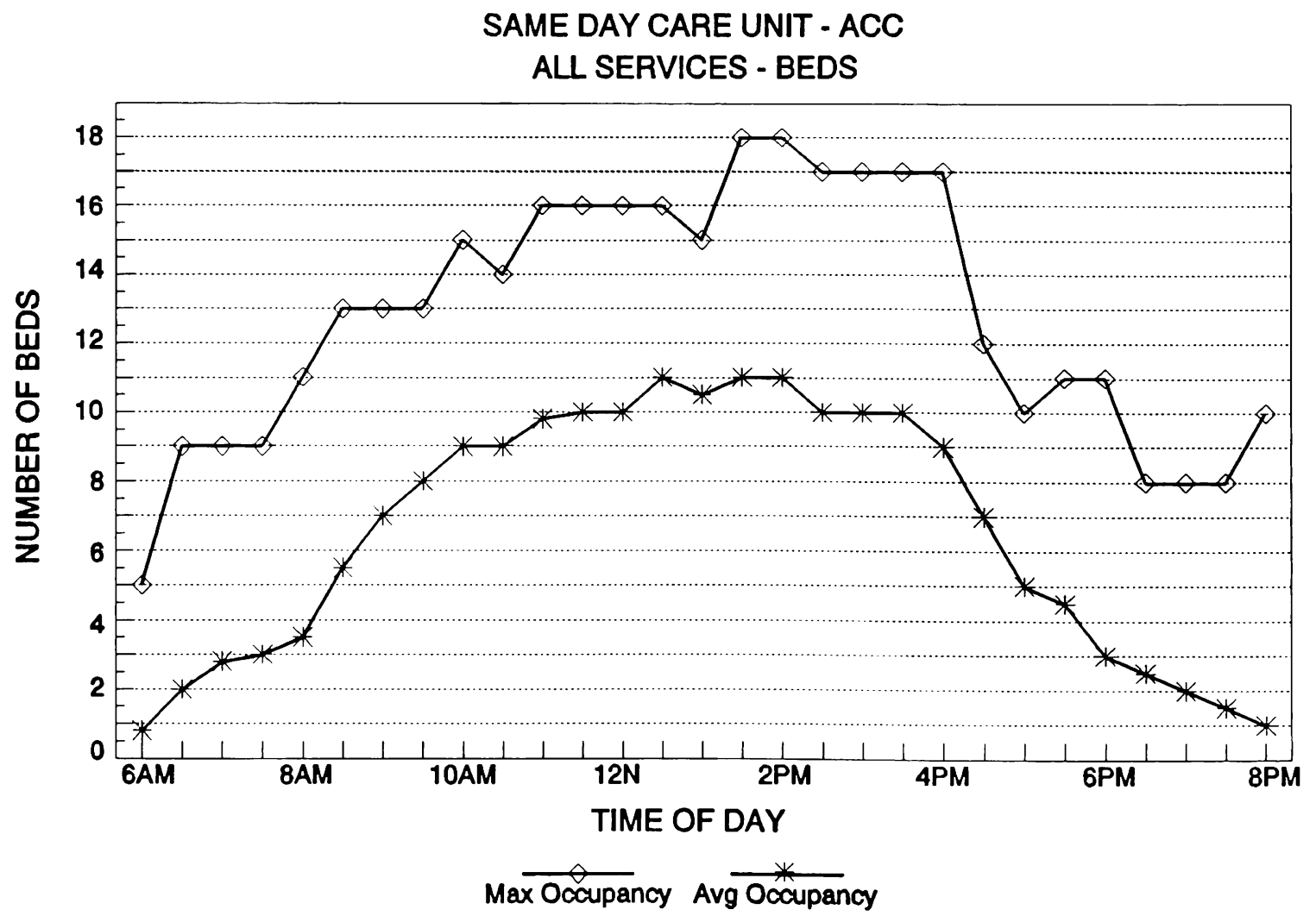

Figure 9: Maximum Number of Beds Required in 100 Simulated Mondays Under Current Conditions 